Research Article

\title{
Evaluation of Severity of Infectious Pneumonia for Newborn Using Ultrasound Image under Adaptive Image Denoising Algorithm
}

\author{
Jieqiong Liu $\mathbb{D},{ }^{1}$ Tingting Lei $\mathbb{D},{ }^{2}$ and Fengyun $\mathrm{Wu} \mathbb{D}^{2}$ \\ ${ }^{1}$ Children's Hospital of Shanxi Province (Shanxi Maternal and Child Health Care Hospital), Taiyuan 030013, Shanxi, China \\ ${ }^{2}$ Department of Ultrasound, East Hospital, Qingdao Municipal Hospital, Qingdao 266000, Shandong, China
}

Correspondence should be addressed to Fengyun Wu; 1530506082@stu.suda.edu.cn

Received 12 July 2021; Revised 15 August 2021; Accepted 16 August 2021; Published 1 September 2021

Academic Editor: Gustavo Ramirez

Copyright (c) 2021 Jieqiong Liu et al. This is an open access article distributed under the Creative Commons Attribution License, which permits unrestricted use, distribution, and reproduction in any medium, provided the original work is properly cited.

\begin{abstract}
This study was to analyze the ultrasound imaging characteristics of infectious pneumonia of newborn in different conditions and the differences in neurobehavioral development. An adaptive image denoising (AID) algorithm was constructed based on multiscale wavelet features. It was compared with the transform domain denoising (TDD) algorithm and spatial domain denoising (SDD) algorithm and applied to ultrasound images of newborns with infectious pneumonia. It was found that the peak signal-to-noise ratio (PSNR), structural similarity (SSIM), and feature similarity index (FSIM) of the constructed algorithm were higher than those of the TDD and SDD algorithms $(P<0.05)$. The ultrasound scores of newborns in noncritical group (group A, $1.54 \pm 0.62$ scores) were all lower than those of the critical group (group B, $3.96 \pm 0.41$ scores) and extremely critical group (group C, $4.25 \pm 0.35$ scores $)(P<0.05)$. The behavioral ability, passive muscle tension, active muscle tension, and original reflection of the newborns in group A were better than other groups $(P<0.05)$. It indicated that the constructed algorithm showed better denoising effect on ultrasound images, which could effectively evaluate the severity of newborns' infectious pneumonia.
\end{abstract}

\section{Introduction}

Infectious pneumonia is a common disease of newborns and also the most common form of newborn infections and an important cause of death. Statistics shows that the death rate of newborn from infectious pneumonia during the perinatal period is about $15 \%[1,2]$. It is mainly induced by different pathogens such as bacteria, viruses, protozoa, and fungi. It can occur before, during, or after childbirth. Its common symptoms include prenatal infectious pneumonia in pregnant women, suffocation, rapid breathing after resuscitation, unstable body temperature, and even respiratory failure in severe cases [3]. However, in actual clinical observations, the symptoms of newborns with infectious pneumonia are very atypical compared with adults and children. The main reason is that the newborn's respiratory system and immune system are not mature enough to show the symptoms of adults or children $[4,5]$. Therefore, it is urgent to find a diagnostic method with high coincidence rate. If it cannot be detected in time and treated with anti-infective treatment, then the sick newborn is very likely to be complicated by persistent pulmonary hypertension, heart enlargement, liver enlargement, and other heart failure manifestations. Children with severe meconium aspiration and acute hypoxia may also have central nervous system symptoms such as disturbance of consciousness, increased intracranial pressure, convulsions, increased red blood cell, hypoglycemia, and hypocalcemia [6].

Currently, bedside X-ray, lung ultrasound, and computerized tomography (CT) are mainly used to diagnose newborn pneumonia. The bedside X-ray is simple to operate and low in price, but it is easily restricted by the position of the newborn, so that the obtained images are prone to multi-tissue overlapping projections. Therefore, the overall quality is low, which brings great adverse effects on evaluation of infectious pneumonia $[7,8]$. CT is the gold standard for infectious pneumonia. It shows the advantages of clear lesion display and 
noninvasiveness. However, the large radiation dose is not suitable for children with critically ill pneumonia. Bedside lung ultrasound is excellent in the diagnosis of newborn pneumonia. It has many advantages such as easy mobility, noninvasiveness, flexible acoustic window, and low cost. It is suitable for multiple examinations of pneumonia in a short period of time $[9,10]$. Adaptive image denoising (AID) algorithm means that, in the process of processing and analysis, the processing method, processing sequence, processing parameters, boundary conditions, or constraint conditions are automatically adjusted according to the data characteristics of the processed data to make it consistent with the statistical distribution characteristics of the processed data. The structural characteristics are adapted to achieve the best processing effect [11]. In the field of medical imaging, AID can be used to process the original image to make the quality of the image more excellent [12]. Therefore, AID was adopted in this study for denoising of ultrasound images of newborn pneumonia in order to provide help in its diagnosis.

In summary, an AID algorithm was constructed herein based on multiscale wavelet features. It was compared with the transform domain denoising (TDD) algorithm and spatial domain denoising (SDD) algorithm and applied to ultrasound images of newborn with infectious pneumonia. The ultrasound scores and neurobehavioral development scores of newborns in group A, group B, group C, and control group (group Ctrl) were compared, aiming to comprehensively explore the ultrasound imaging characteristics of infectious pneumonia of newborn in different conditions and differences in neurobehavioral development.

\section{Materials and Methods}

2.1. Selection of Research Objects. 98 newborns with infectious pneumonia in hospital from May 5, 2019, to April 20, 2020, were selected and included in the experimental group. There were 47 males and 51 females, with the age of 1-30 days. In addition, 45 newborns with jaundice who came to the hospital during the same period were selected and included in the group C. There were 25 males and 20 females, with the age of 1-30 days. The newborns in experimental group were grouped into a noncritical group (group A), a critical group (group B), and an extremely critical group (group C) based on the severity evaluated by lung ultrasound score (LUS). The study had been approved by the medical ethics committee of the hospital, and the newborn family members had learned about the study and signed the informed consent forms.

The inclusion criteria were defined as follows: patients with complete basic clinical data; patients who had not received drug treatment; newborns with patchy shadows on $\mathrm{X}$-ray films that may be accompanied by atelectasis; and newborns not crying at birth but fast breathing after resuscitation.

The exclusion criteria were defined as follows: newborn with no clear evidence of infection, newborn with heart disease, newborn with pneumothorax, and newborn with meconium aspiration syndrome.
2.2. Lung Ultrasound. The GE LOGIQ E portable Doppler ultrasound system (from United States) was adopted to scan newborns with infectious pneumonia with a convex abdomen probe at a frequency of $6.0 \mathrm{MHz}$. The newborn was placed in the supine position and the front chest wall, the side chest walls, and back chest wall were scanned based on the 12-lung-area division method. Both sides of the chest wall were scanned from top to bottom and from the inside to the outside. It should be scanned being perpendicular to the intercostal space, the probe was perpendicular to the chest wall, with the sign of "bat sign," and then it was rotated $90^{\circ}$ to scan along the intercostal space. After the scan was completed, the acquired ultrasound images were sent to the workstation for processing, and two senior imaging physicians were required for independent diagnosis jointly.

Normal lung was manifested as follows: the lung tissue was hypoechoic; the pleural line was complete, smooth, clear, and regular, with high echoic bright lines; the thickness of the pleura was less than $5 \mathrm{~cm}$; and multiple A-lines were parallel to the pleural line.

Abnormal lung was manifested as follows: multiple B-lines can be seen during longitudinal cross-sectional scan, which is interpreted as interstitial pulmonary syndrome; there is an irregular hypoechoic area under the pleura, and the short-line-like strong echo movement amplitude exceeds $1 \mathrm{~mm}$ during inhalation, which is interpreted as lung consolidation; A-line disappears; the pleural line is thickened, blurred, interrupted, or not smooth, with enhanced echo; pleural effusion and lung slip are reduced. Ultrasound imaging of the patient's lungs was evaluated with the LUS.

\subsection{AID Algorithm Based on Multiscale Wavelet Features.} Various defects such as artifacts and noise that were not conducive to evaluation could be generated during the scanning due to various objective factors. Therefore, choosing a suitable method to process the image to improve the overall quality of the image is a hot research topic. The theory of multiscale wavelet features [13] was introduced herein to extract and characterize the wavelet features of the original image. The algorithm was mainly divided into three steps: noise type detection, noise level prediction, and adaptive noise reduction. Under different noise levels, different noise types were applied to the normal image to extract their feature vectors; then a set of learning data could be obtained:

$$
\left\{\left(P_{1}^{Q_{i}}, Q_{i}\right), \ldots,\left(P_{j}^{Q_{i}}, Q_{i}\right)\right\} \in U^{Q_{i}} * R
$$

where $Q_{i}$ represents the set of noise types, complying with $Q_{i} \in\left[Q_{x}, Q_{y}, Q_{z}\right], Q_{x}$ represents the Gaussian noise, $Q_{y}$ refers to the impulse noise, and $Q_{z}$ represents the Gaussian white noise under mixed noise. $U^{Q_{i}}, P_{j}{ }_{i}$, and $R$ represent the feature vector space, feature vector, and noise level, respectively. Then, the acquired learning data was applied to construct a noise classification algorithm:

$$
\theta(P)=\langle\vartheta, P\rangle+a
$$


In equation (2), $a \in R$ and $\vartheta \in P^{Q_{i}}$. For the Gaussian noise $Q_{x}$, the different noise standard deviations were applied to the normal image to extract the feature vectors, so as to obtain a set of learning data:

$$
\left\{\left(P_{1}^{Q_{x}}, Q_{x}\right), \ldots,\left(P_{j}^{Q_{x}}, Q_{x}\right)\right\} \in U^{Q_{x}} * R
$$

where $U^{Q_{x}}$ represents the feature vector space of Gaussian noise. Then, this dataset could be adopted to construct an evaluation algorithm of Gaussian noise standard deviation:

$$
\delta_{Q_{x}}(P)=\langle\tau, P\rangle+b .
$$

In equation (4), $b \in R$ and $\tau \in P^{Q_{x}}$. For impulse noise, the different noise pollution ratios were applied to normal images to extract the feature vectors; then a set of learning data also could be obtained:

$$
\left\{\left(P_{1}^{Q_{y}}, Q_{y}\right), \ldots,\left(P_{j}^{Q_{y}}, Q_{y}\right)\right\} \in U^{Q_{y}} * R,
$$

where $U^{Q_{y}}$ represents the feature vector space under impulse noise. An evaluation algorithm for impulse noise pollution ratio could be constructed using the learning data:

$$
\delta_{Q_{y}}(P)=\langle\xi, P\rangle+c .
$$

In equation (6), $c \in R$ and $\xi \in P^{Q_{x}}$. Similarly, the available learning data and evaluation model were given as follows for Gaussian white noise under mixed noise:

$$
\begin{aligned}
& \left\{\left(P_{1}^{Q_{z}}, Q_{z}\right), \ldots,\left(P_{j}^{Q_{z}}, Q_{z}\right)\right\} \in U^{Q_{z}} * R, \\
& \delta_{Q_{z}}(P)=\langle\psi, P\rangle+d .
\end{aligned}
$$

In equations (7) and (8), $U^{Q_{z}}$ represents the feature vector space of Gaussian white noise under the mixed noise, $d \in R$ and $\psi \in P^{Q_{x}}$. The variance of Gaussian noise was an important parameter in the process of image noise reduction, but the value was unknown. The abovementioned algorithm can accurately predict the variance of Gaussian noise to obtain a parameter adaptive noise reduction algorithm. The same proportion of salt and pepper noise pollution will have an impact on the noise reduction effect, and the optimized AID algorithm can be obtained after accurate values are obtained.

2.4. Evaluation Indicator of the Algorithm. The TDD [14] and SDD [15] were incorporated to compare with the AID algorithm proposed. The indicators to evaluate the algorithm's denoising performance included peak signal-to-noise ratio (PSNR), structural similarity (SSIM), and feature similarity index (FSIM).

(1) PSNR: it could indicate the signal difference between two images, expressed as follows:

$$
\begin{aligned}
\mathrm{PSNR} & =10 \log _{10} \cdot \frac{2^{p}-1}{\mathrm{MSE}}, \\
\mathrm{MSE} & =\sqrt{\frac{1}{U * I * L} \sum_{i=1}^{U} \sum_{j=1}^{I} \sum_{z=1}^{L}\left(r^{w}(i, j)-v^{w}(i, j)\right)^{2}},
\end{aligned}
$$

where $p$ represents the $p$ bits quantized image, $U * I$ represents the size of the image, $L$ refers to the number of channels of the image, $r^{w}(i, j)$ represents the position of the processed image at channel $w$, and $v^{w}(i, j)$ represents the position of the original image at channel $w$.

(2) SSIM: it could not only refer to the signal difference, but also refer to the structure information of the image, expressed as follows:

$$
\begin{aligned}
\operatorname{SSIM} & =\frac{\sum_{j-1}^{m} Q\left(x_{i}, j_{i}\right) \cdot P\left(x_{i}, j_{i}\right)}{\sum_{j-1}^{m} Q\left(x_{i}, j_{i}\right)}, \\
P\left(x_{i}, j_{i}\right) & =[a(x, y)]^{\eta} *[b(x, y)]^{\theta} *[c(x, y)]^{\lambda} .
\end{aligned}
$$

where $m$ represents the number of windows, $Q\left(x_{i}, j_{i}\right)$ represents the weight of the SSIM, and $P\left(x_{i}, j_{i}\right)$ represents the value of the SSIM. $\eta, \theta$, and $\lambda$ were all weight coefficients, $a(x, y)$ represents the brightness of the image, $b(x, y)$ refers to the contrast of the image, and $c(x, y)$ indicates the structure of the image.

(3) FSIM: evaluation based on image features can be expressed as follows:

$$
\begin{aligned}
\text { FSIM } & =\frac{\sum_{X \in \Omega} W(X) \cdot\left[W_{C}(X)\right]^{\varepsilon} M C_{h}(X)}{\sum_{X \in \sigma} M C_{h}(X)}, \\
W(X) & =\left[W_{M C}(X)\right]^{\kappa} \cdot\left[W_{T}(X)\right]^{\vartheta}, \\
W_{M C}(X) & =\frac{2 M C_{1}(X) \cdot M C_{2}(X)+H_{1}}{M C_{1}^{2}(X)+M C_{2}^{2}(X)+H_{1}}, \\
W_{T}(X) & =\frac{2 T_{1}(X) \cdot T_{2}(X)+H_{2}}{P T_{1}^{2}(X)+P T_{2}^{2}(X)+H_{2}} .
\end{aligned}
$$

In equations (13)-(16), $\Omega$ represents the image space, $\kappa$ and $\vartheta$ are weight coefficients, and $\varepsilon$ refers to the chromaticity factor. $W_{M C}(X), W_{T}(X)$, and $W$ represent the phase similarity, gradient amplitude similarity, and phase consistency feature equation, respectively. $T$ refers to the gradient amplitude characteristic equation, and $H_{1}$ and $H_{2}$ are constants.

2.5. Observation Indicators. The age, weight, gestational age, number of full-term, and premature babies of the newborns were counted and recorded in the two groups. The score on the newborns in critical case was recorded in the experimental group. The LUSs of group A, group B, group C, and group Ctrl were compared and analyzed. Neonatal behavioral neurological assessment (NBNA) was used to evaluate the degree of newborn neurobehavioral development, including behavioral ability, passive muscle tension, active muscle tension, original reflection, and general reflection. 
2.6. Statistical Methods. The data were analyzed and processed by SPSS19.0 version statistical software, the measurement data were expressed as mean \pm standard deviation $(\bar{x} \pm s)$, and the count data were shown with percentage. The difference was statistically significant at $P<0.05$.

\section{Results}

3.1. Comparison on Denoising Performances of Three Algorithms. Figure 1 shows the comparisons of SSIM and FSIM of the three algorithms, and Figure 2 illustrates the comparison of PSNR level of the three algorithms. The SSIM of TDD, SDD, and AID was $0.77 \pm 0.08,0.73 \pm 0.12$, and $0.95 \pm 0.07$, respectively; the FSIM of TDD, SDD, and AID was $0.82 \pm 0.13,0.85 \pm 0.08$, and $0.94 \pm 0.11$, respectively; and the PSNR of TDD, SDD, and AID was 40.11 \pm 7.23 , $38.94 \pm 3.29$, and $49.56 \pm 4.05$, respectively. It was clear that the SSIM, FSIM, and PSNR of the AID algorithm were obviously higher than those of TDD and SDD algorithms $(P<0.05)$, and those between the TDD algorithm and SDD algorithm were not visibly different from each other $(P>0.05)$.

3.2. Comparison on Basic Data of Two Groups of Newborns. Figure 3 shows the comparison of the age, weight, and gestational age of newborns in the two groups. It indicated that age of the newborn in the experimental group and the group Ctrl was $17.54 \pm 6.05$ days and $19.33 \pm 7.26$ days, respectively; the weight in experimental group and group Ctrl was $3.26 \pm 0.75 \mathrm{~kg}$ and $3.11 \pm 0.58 \mathrm{~kg}$, respectively; and the gestational age was $38.96 \pm 7.45$ weeks and $38.04 \pm 9.11$ weeks, respectively. Therefore, the age, weight, and gestational age of newborns in the two groups were not observably different from each other $(P>0.05)$.

Figure 4 illustrates the comparison results of the proportions of full-term newborns and preterm infants between the two groups. The proportions of full-term newborns and preterm infants in the experimental group were $65.34 \%$ and $34.66 \%$, respectively; those in the group Ctrl were $81.23 \%$ and $18.77 \%$, respectively. Thus, the experimental group showed lower proportion of full-term newborn and higher proportion of premature infants, and the difference was extremely different $(P>0.05)$.

3.3. Lung Ultrasound Findings of Two Groups of Newborns. Figure 5 shows an ultrasound image of lung of a male newborn in the experimental group (aged 15 days). The pleural line was thickened, fuzzy, and irregular, there was an irregular hypoechoic area under the lung pleura, the movement range of short-line strong echoes exceeded $1 \mathrm{~mm}$ during inhalation, there were more than $3 \mathrm{~B}$-lines in the field, and the A-line was not visible. Thus, it could be judged as pulmonary parenchymal disease with bronchial inflation. Figure 6 shows an ultrasound image of a male newborn in group Ctrl. There was a high echo line that slid back and forth with the breathing motion in the depth of the rib line, which was the pleural line. When the ultrasound was projected perpendicularly to the surface of the pleura-lung,

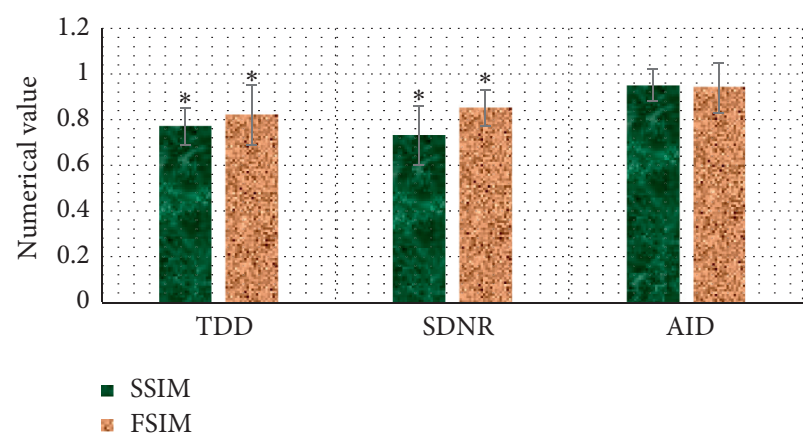

FIgURE 1: Comparison on SSIM and FSIM of three algorithms. Note: ${ }^{*}$ indicates the difference was visible in contrast to the AID algorithm $(P<0.05)$.

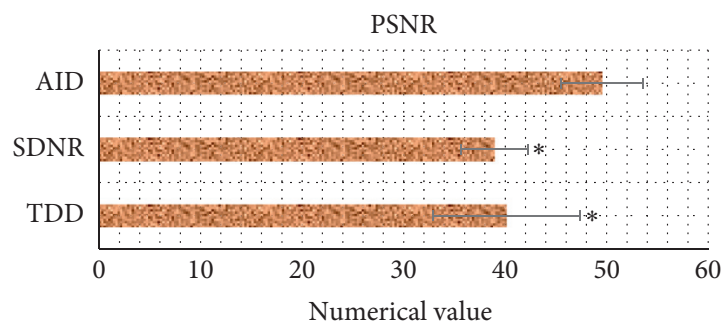

FIGURE 2: Comparison on PSNR level of the three algorithms. Note: * indicates the difference was visible in contrast to the AID algorithm $(P<0.05)$.

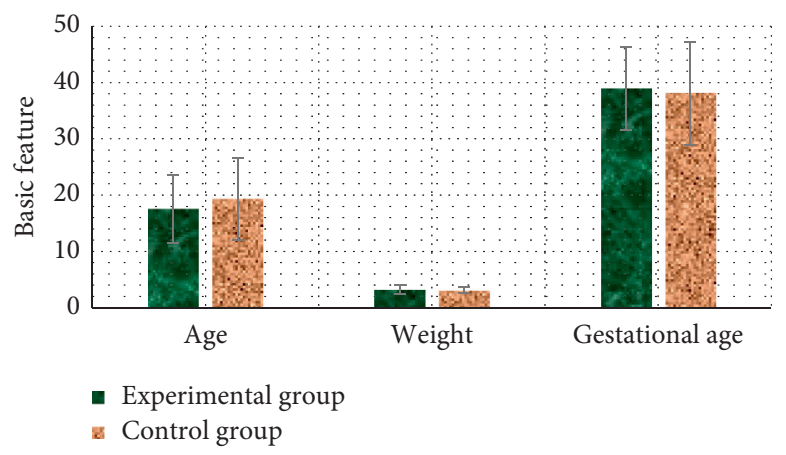

FIgURE 3: Comparison on the age, weight, and gestational age of newborns in the two groups.

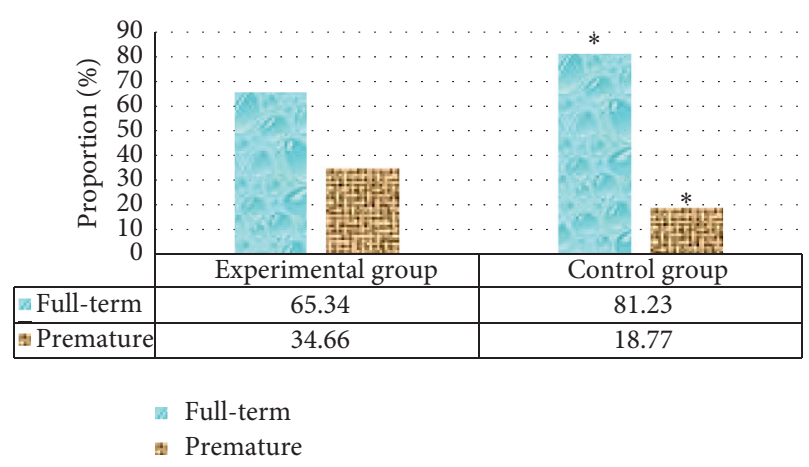

FIgURE 4: Comparison results of the proportions of full-term newborns and preterm infants. 


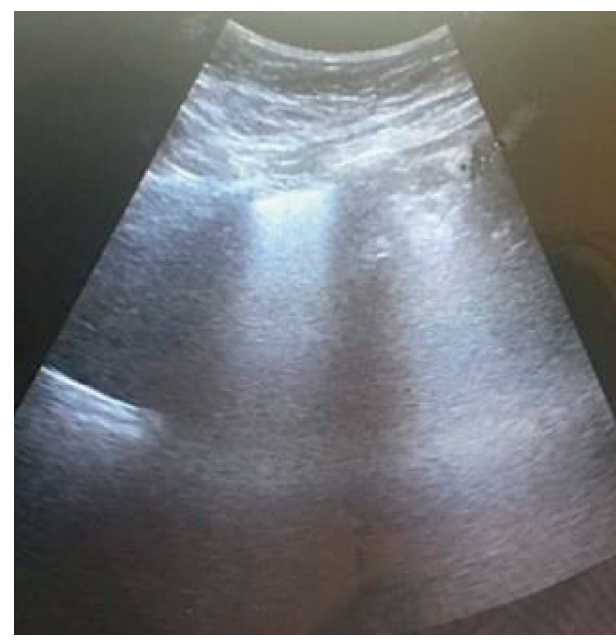

Figure 5: An ultrasound image of lung of a male newborn in the experimental group (aged 15 days).

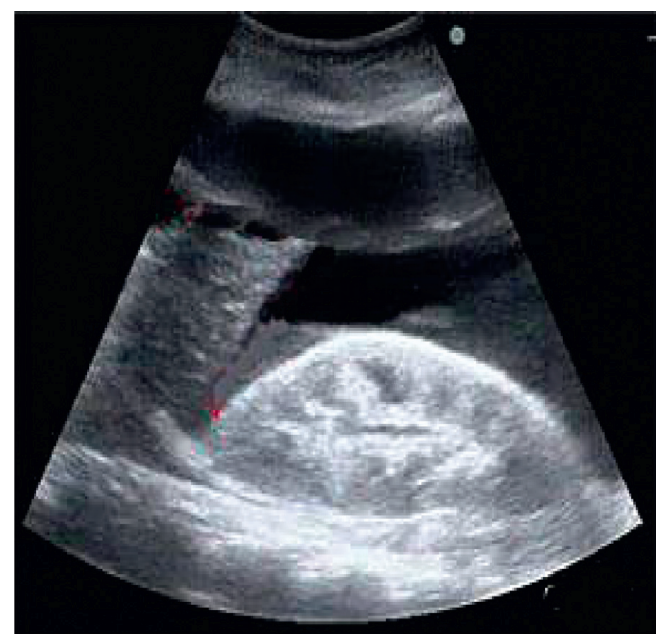

Figure 6: An ultrasound image of a male newborn in group Ctrl (aged 21 days).

reverberation artifacts may appear, manifested as multiple echoes arranged at equal distances, and their intensity decreased successively, which was the A-line.

\subsection{Score of Newborn in Critical Cases in the Experimental} Group. Figure 7 illustrates the scores of newborn in critical cases in the experimental group. It indicated that there were 17 cases with $\leq 70$ scores, 34 cases with $71-90$ scores, and 48 cases with scores of $>90$. Therefore, they were divided into the extremely critical group (group A), critical group (group $\mathrm{B}$ ), and noncritical group (group C) accordingly.

3.5. Comparison on LUS of Newborns in Group C, Group B, Group A, and Group Ctrl. The LUSs of newborns in groups $\mathrm{C}, \mathrm{B}, \mathrm{A}$, and Ctrl were compared, and the results are illustrated in Figure 8. It revealed that the LUSs in groups C, B, $A$, and Ctrl were $4.25 \pm 0.35$ scores, $3.96 \pm 0.41$ scores, $1.54 \pm 0.62$ scores, and $0.28 \pm 0.01$ scores, respectively. Thus, it indicated that the LUS in group $C$ showed no visible difference in contrast to that in group $\mathrm{B}(P>0.05)$; the LUS

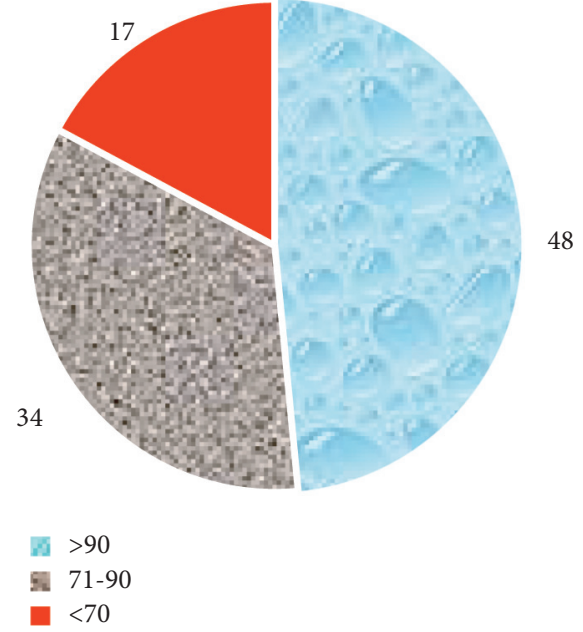

FIGURE 7: Score of newborn in critical cases in the experimental group.

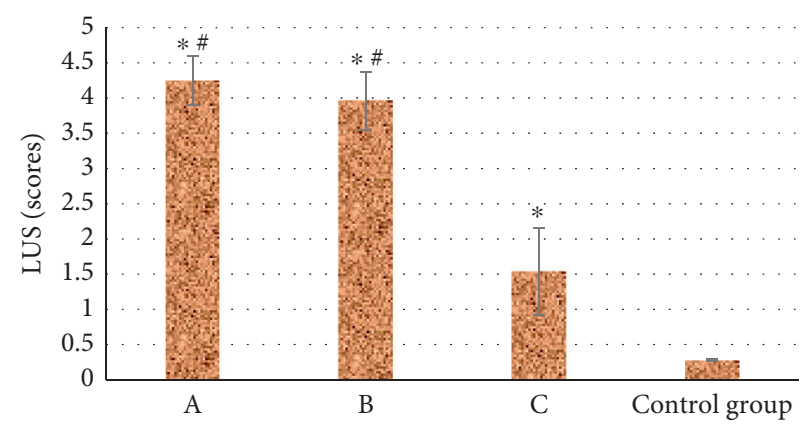

Figure 8: The LUSs of newborns in groups C, B, A, and Ctrl. Note: * suggests the difference was extreme in contrast to group Ctrl $(P<0.05) ;{ }^{\#}$ indicates that there was an extreme difference in contrast to group A $(P<0.05)$.

in group Ctrl was obviously lower in contrast to the other three groups $(P<0.05)$.

3.6. Comparison on Neurobehavioral Development Scores of Newborns in Groups C, B, A, and Ctrl. The neurobehavioral development scores of newborns in groups $\mathrm{C}, \mathrm{B}, \mathrm{A}$, and Ctrl were compared, and the results are illustrated in Figures 9-12. Analysis on the figures showed that the behavioral ability, passive muscle tension, active muscle tension, and original reflection were not different greatly in groups $\mathrm{B}$ and $\mathrm{C}(P>0.05)$; the scores of these four indicators in group Ctrl were observably larger than those in the other three groups $(P<0.05)$; and those in group A were larger greatly in contrast to groups $\mathrm{B}$ and $\mathrm{C}(P<0.05)$.

\section{Discussion}

Due to the imperfect development of the newborn's organs and low immunity, the symptoms of infectious pneumonia of newborn are not typical enough, leading to the inability to detect and treat them in time. Therefore, choosing an 


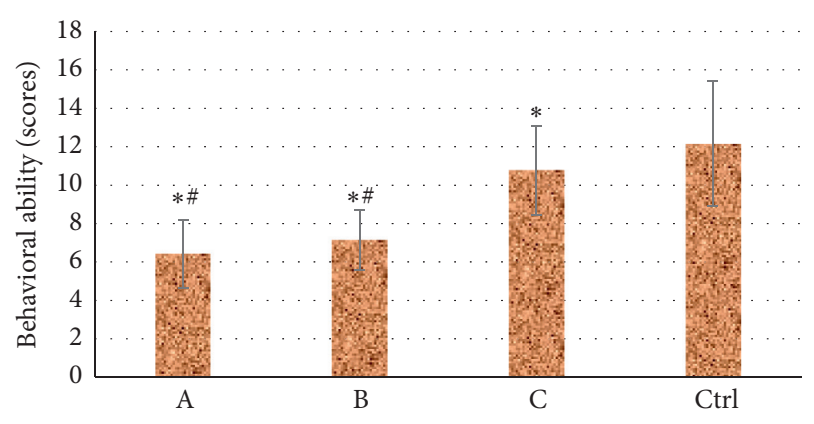

FIgURE 9: Comparison on behavioral ability of newborns in each group. Note: ${ }^{*}$ suggests the difference was extreme in contrast to group Ctrl $(P<0.05)$; ${ }^{\#}$ indicates that there was an extreme difference in contrast to group A $(P<0.05)$.

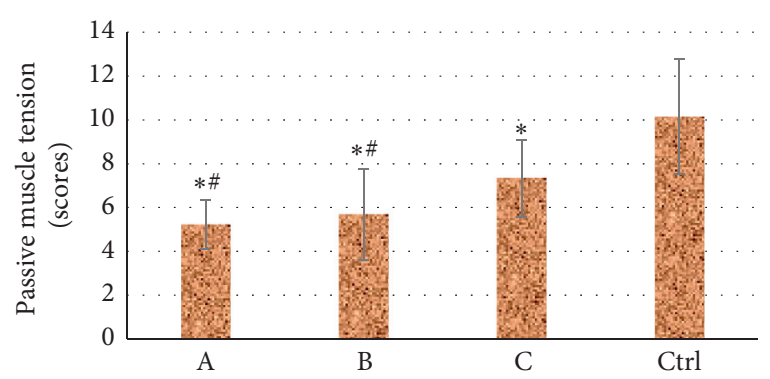

FIgURE 10: Comparison on passive muscle tension of newborns in each group. Note: * suggests the difference was extreme in contrast to group $\mathrm{Ctrl}(P<0.05)$; ${ }^{\#}$ indicates that there was an extreme difference in contrast to group A $(P<0.05)$.

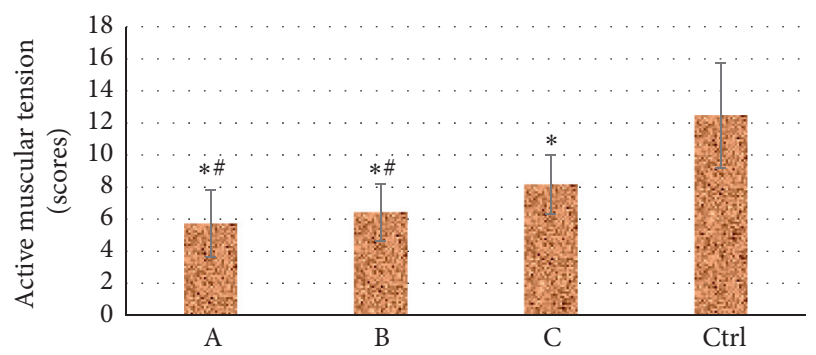

FIgURE 11: Comparison on active muscle tension of newborns in each group. Note: ${ }^{*}$ suggests the difference was extreme in contrast to group Ctrl $(P<0.05)$; ${ }^{\#}$ indicates that there was an extreme difference in contrast to group A $(P<0.05)$.

effective examination method is an urgent problem that needs to be solved in the clinic [16]. Therefore, an AID algorithm was constructed based on multiscale wavelet features and compared with the TDD algorithm and SDD algorithm in this study. In addition, the construction algorithm was applied to the ultrasound image diagnosis of newborns with infectious pneumonia. The results showed that the SSIM, FSIM, and PSNR of the AID algorithm were visibly larger than those of the TDD and SDD algorithms, and the differences were dramatic $(P<0.05)$. Such results were similar to the research results of Gao and Cai [17], indicating that the constructed algorithm based on the multiscale wavelet features showed better performance in

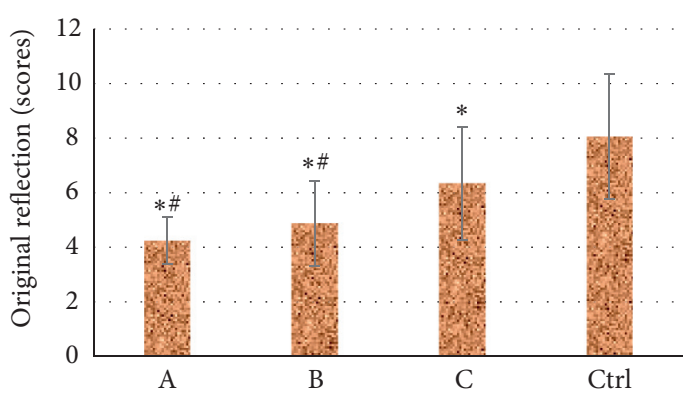

FIGURE 12: Comparison on original reflection of newborns in each group. Note: ${ }^{*}$ suggests the difference was extreme in contrast to group Ctrl $(P<0.05)$; \# indicates that there was an extreme difference in contrast to group A $(P<0.05)$.

denoising ultrasound images. The proportion of newborn full-term infants in the experimental group was much smaller, while the proportion of preterm infants was greater, and the differences were obvious $(P<0.05)$. Preterm infants are more likely to develop infectious pneumonia, which may be related to the delayed development of the system caused by premature.

There were 17 cases with scores of $\leq 70$ scores, 34 cases with 71-90 scores, and 48 cases with scores of $>90$ in the experimental group, so they were divided into the groups $\mathrm{C}$, $\mathrm{B}$, and A accordingly. Further analysis found that the LUS scores for newborns in group Ctrl were lower observably in contrast to the groups $\mathrm{C}, \mathrm{B}$, and $\mathrm{A}$, and the differences were dramatic $(P<0.05)$. It was consistent with the research results of Dolgorsuren et al. [18], suggesting that the LUS of newborns with infectious pneumonia was high. In addition, the LUS scores of newborns in group A were hugely lower in contrast to groups B and C $(P<0.05)$, which also showed that the LUS could effectively distinguish the severity of infectious pneumonia of newborn. The behavioral ability, passive muscle tension, active muscle tension, and original reflection of newborns in group Ctrl were all greater much than those in the other three groups $(P<0.05)$. Such findings indicated that the neurobehavioral development of infectious pneumonia in newborn was worse than healthy newborn with lower indicators. The behavioral ability, passive muscle tension, active muscle tension, and original reflection for newborns in group A were found to be larger than groups $\mathrm{B}$ and $\mathrm{C}$, and the differences were remarkable $(P<0.05)$, which was similar to the results of Qiu et al. [19]. It suggested that, with the aggravation of newborn pneumonia, the neurobehavioral development of newborn gets worse.

\section{Conclusion}

An AID algorithm was constructed based on multiscale wavelet features, compared with the TDD algorithm and SDD algorithm, and applied to the ultrasound image diagnosis of newborns with infectious pneumonia. The results showed that the algorithm proposed was better in denoising ultrasound images. Ultrasound images could effectively assess the severity of infectious pneumonia of newborn. As 
the disease worsened, the newborn's neurobehavioral development became worse. However, it only discussed infectious pneumonia of newborn and failed to involve other lung lesions. In addition, the differences in ultrasound imaging of different pneumonias were not investigated in depth. Thus, it will consider increasing the selection of newborn samples and further analyze the value of ultrasound in pneumonia of newborn. In conclusion, this study may provide a new evaluation method for the clinical diagnosis of neonatal infectious pneumonia.

\section{Data Availability}

The data used to support the findings of this study are available from the corresponding author upon request.

\section{Conflicts of Interest}

The authors declare no conflicts of interest.

\section{References}

[1] N. Sauwen, M. Acou, S. Van Cauter et al., "Comparison of unsupervised classification methods for brain tumor segmentation using multi-parametric MRI," NeuroImage-Clinical, vol. 12, pp. 753-764, 2016.

[2] F. Özyurt, E. Sert, and D. Avc1, "An expert system for brain tumor detection: fuzzy C-means with super resolution and convolutional neural network with extreme learning machine," Medical Hypotheses, vol. 134, Article ID 109433, 2020.

[3] J. E. Park, H. S. Kim, S. J. Kim, J. H. Kim, and W. H. Shim, "Alteration of long-distance functional connectivity and network topology in patients with supratentorial gliomas," Neuroradiology, vol. 58, no. 3, pp. 311-320, 2016.

[4] F. Şişik and E. Sert, "Brain tumor segmentation approach based on the extreme learning machine and significantly fast and robust fuzzy C-means clustering algorithms running on Raspberry Pi hardware," Medical Hypotheses, vol. 136, Article ID 109507, 2020.

[5] S. N. Kumar, A. L. Fred, and P. S. Varghese, "Suspicious lesion segmentation on brain, mammograms and breast MR images using new optimized spatial feature based super-pixel fuzzy c-means clustering," Journal of Digital Imaging, vol. 2, no. 2, pp. 322-335, 2019.

[6] A. Geetha and N. Gomathi, "A robust grey wolf-based deep learning for brain tumour detection in MR images," Biomedizinische Technik, vol. 65, no. 2, pp. 191-207, 2020.

[7] H. W. Goo and Y. S. Ra, "Advanced MRI for pediatric brain tumors with emphasis on clinical benefits," Korean Journal of Radiology, vol. 18, no. 1, pp. 194-207, 2017.

[8] O. Shearkhani, A. Khademi, A. Eilaghi et al., "Detection of volume-changing metastatic brain tumors on longitudinal MRI using a semiautomated algorithm based on the jacobian operator field," American Journal of Neuroradiology, vol. 38, no. 11, pp. 2059-2066, 2017.

[9] J. H. Hara, A. Wu, J. E. Villanueva-Meyer et al., "Clinical applications of quantitative 3-dimensional MRI analysis for pediatric embryonal brain tumors," International Journal of Radiation Oncology, Biology, Physics, vol. 102, no. 4, pp. 744-756, 2018.

[10] T. Jin, B. Iordanova, T. K. Hitchens et al., "Chemical exchange-sensitive spin-lock (CESL) MRI of glucose and analogs in brain tumors," Magnetic Resonance in Medicine, vol. 80, no. 2, pp. 488-495, 2018.

[11] Z. Lv, X. Li, H. Lv, and W. Xiu, "BIM big data storage in WebVRGIS," IEEE Transactions on Industrial Informatics, vol. 16, no. 99, p. 1, 2019.

[12] M. Spanakis, E. Kontopodis, S. Van Cauter, V Sakkalis, and $\mathrm{K}$ Marias, "Assessment of DCE-MRI parameters for brain tumors through implementation of physiologically-based pharmacokinetic model approaches for Gd-DOTA," Journal of Pharmacokinetics and Pharmacodynamics, vol. 43, no. 5, pp. 529-547, 2016.

[13] Y. Chen, S. Hu, H. Mao, W Deng, and X Gao, "Application of the best evacuation model of deep learning in the design of public structures," Image and Vision Computing, vol. 102, Article ID 103975, 2020.

[14] A. Chakhoyan, C. Raymond, J. Chen et al., "Probabilistic independent component analysis of dynamic susceptibility contrast perfusion MRI in metastatic brain tumors," Cancer Imaging, vol. 19, no. 1, p. 14, 2019.

[15] R. Morabito, C. Alafaci, S. Pergolizzi et al., "DCE and DSC perfusion MRI diagnostic accuracy in the follow-up of primary and metastatic intra-axial brain tumors treated by radiosurgery with cyberknife," Radiation Oncology, vol. 14, no. 1, p. 65, 2019.

[16] C. Su, L. Zhao, S. Li et al., "Amid proton transfer (APT) and magnetization transfer (MT) MRI contrasts provide complimentary assessment of brain tumors similarly to proton magnetic resonance spectroscopy imaging (MRSI)," European Radiology, vol. 29, no. 3, pp. 1203-1210, 2019.

[17] X. Gao and J. Cai, "Optimization analysis of urban function regional planning based on big data and GIS Technology," Boletin Tecnico/Technical Bulletin, vol. 55, no. 11, pp. 344-351, 2017.

[18] E. A. Dolgorsuren, M. Harada, Y. Kanazawa et al., "Correlation and characteristics of intravoxel incoherent motion and arterial spin labeling techniques versus multiple parameters obtained on dynamic susceptibility contrast perfusion MRI for brain tumors," Journal of Medical Investigation, vol. 66, no. 3, pp. 308-313, 2019.

[19] R. X. Qiu, X. Liu, J. L. Wang et al., “Clinical effect of exogenous pulmonary surfactant in the treatment of severe neonatal infectious pneumonia: a multicenter prospective clinical trial," Zhong Guo Dang Dai Er Ke Za Zhi, vol. 21, no. 4, pp. 327-331, 2019. 